\section{The Bright Future of Digital Imaging in Scanning Electron Microscopy}

\author{
M.T. Postek \& A.E. Vladar*, NIST, Gaithersburg, MD
}

One of the major advancements applied to scanning electron microscopy (SEM) during the past 10 years has been the development and application of digital imaging technology. Advancements in technology, notably the availability of less expensive, high-density memory chips and the development of high speed analog-to-digital converters, mass storage and high performance central processing units have fostered this revolution. Today, most modern SEM instruments have digital electronics as a standard feature. These instruments, generally have 8 bit or 256 gray levels with, at least, $512 \times 512$ pixel density operating at TV rate. In addition, current slow-scan commercial frame-grabber cards, directly applicable to the SEM, can have upwards of 12-14 bit lateral resolution permitting image acquisition at $4096 \times 4096$ resolution or greater. The two major categories of SEM systems to which digital technology have been applied are:

1. Analog "slow scan" SEM systems. In the analog SEM system the scan generator is normally operated in an analog manner and the image is displayed in an analog or "slow scan" mode. TV rate is available, but is not the preferred mode of operation. These systems have been the mainstay of the SEM market and many thousands of instruments are currently out in the field. Analog systems are not directly or economically upgradable by the SEM manufacturer to digital imaging, but is is possible to install accessory digital image processing and analysis systems such as those provided by third-party companies. These systems include digital imaging incorporated into $x$-ray microanalysis systems or other computer-based stand alone systems. External access of the system is through a port referred to as a digital beam interface $(\mathrm{DBl})$. The $\mathrm{DB}$ l changes the voltage levels, signal polarities,
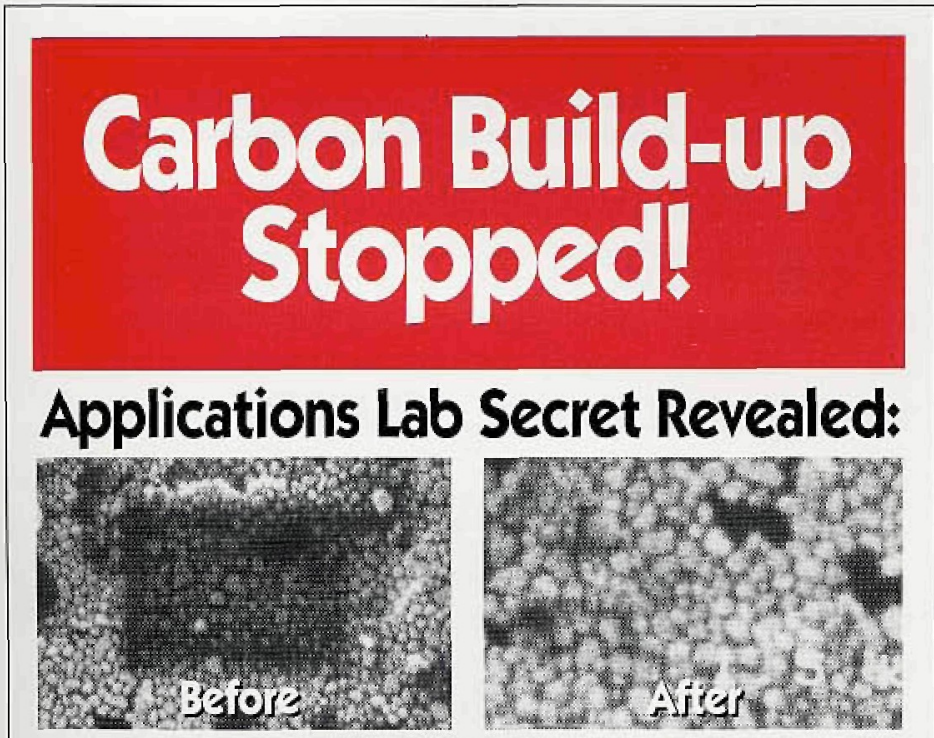

SEM manufacturers won't admit it, but most SEMs are subject to contamination build-up-even dry pumped systems. To stop hydrocarbon condensation, major applications labs and SEM users rely on the XEI Scientific SEM-CLEAN ${ }^{\prime M}$ system.

Result: Outstanding pictures at low $\mathrm{kV}$ and high resolution and no oil on EDS X-ray detector windows. The Nitrogen purge of the inexpensive SEM-CLEAN system cleans your electron microscope while you're away.

\section{SEM-CLEAN"' Stops the Oil}

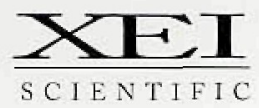

3124 Wessex Way, Redwood City, CA 94061 - 415-369-0133 - Fax 415-363-1659 biases and impedances in order to properly connect the standard inputs and outputs of the analog and digital converters of the external computer to the SEM. This port is either standard on the instrument or it can be added later.

2. Digital SEM systems. In the digital SEM system, the scan generator is operated either in an analog or digital manner. The image is stored, processed and displayed transparently through an image memory routinely at TV rate. Internal or external hard-disk mass storage of the digital images is also possible for archiving. The stored digital image may be photographed directly from the frame memory or hard disk. High resolution photography is accomplished through direct analog or digital scanning. High-speed frame-grabbing is also possible with an accessory computer through a standard video out put (RS-170 or PAL). External communication to other third party systems is accomplished through a DBI or other standard communication channels such as the Small Computer Standard Interface (SCSI).

Pre-digital electronics SEMs were plagued by the problem of a poor signalto-noise ratio, especially at low accelerating voltages and TV scan rates. Recent developments in field emission filament technology improved that situation, but parallel development of the modern digital imaging technology brought both of these technologies together into an extremely powerful tool with exceptional flexibility. Some of the advantages afforded by digital imaging include:

TV Rate Scanning. Essentially the "slow scan" presentation of the SEM is gone and is replaced with a real-time TV image. Integration of poorer signal-to-noise images is tansparently accomplished by frame buffering and frame averaging of the video signal. TV rate scanning has been shown to be useful in reduction of charging on many samples.

Digital Image Storage. Image archiving of the digital images either to floppy disk or hard disk provides a permanent record that is easy to retrieve. Image quality is identical to the originally stored image. Standardized file storage such as the TIFF file format enables importation of the images into desk-top computers and word processing programs.

Paperless Image Transmission. The image data can be transmitted via data lines to remote locations. It is conceptually possible to view the SEM image from a remote location and actually operate the SEM from that location.

Real-time Pseudocolorization. High speed frame grabbers built into fast computers enable the pseudocolorization of the SEM image at TV rate. This allows the highlighting by color coding of certain information valuable to the viewer.

Real-time Image Analysis/Processing. Digital enhancement of the image can be done transparently, as the image is acquired, and the image can be processed at the SEM console.

Optimization of Operating Conditions. Digital SEMs can automatically optimize the operating conditions, such as the brightness, contrast, focus and astigmatism correction. The operator can save optimum operating conditions then re-load them as needed.

Until a few years ago, digital imaging was severely limited by the power of the computer systems available and, therefore much of the digital imaging was done through interfacing to the powerful mini-computer coupled to an x-ray microanalysis systems. Today, many desktop computers have computing capabilities surpassing these early mini-computers. Computer systems are now small and inexpensive enough to be directly incorporated into the SEM electronics console as a standard component by the manufacturer. Thus, the architecture of the modern SEM has changed, in that, the instrument has become more a computer with an SEM column being only a peripheral component. This concept is a major acvantage because the digital architecture of modern SEMs now permits the application of a whole host of peripheral technologies associated with, and being developed for, the personal computer industry to be readily applied to the SEM. These technologies enable the data to be more readily taken, stored and processed. Indeed, the future is bright for this technology.

* Guest Researcher from the Hungarian Academy of Sciences.

Reprinted from Proceedings, 51st Meeting of the Microscopy Society of America with the permission of MSA 


\section{Power up your}

\section{x-ray microdnalysis with...}

\section{PowerMx TM}

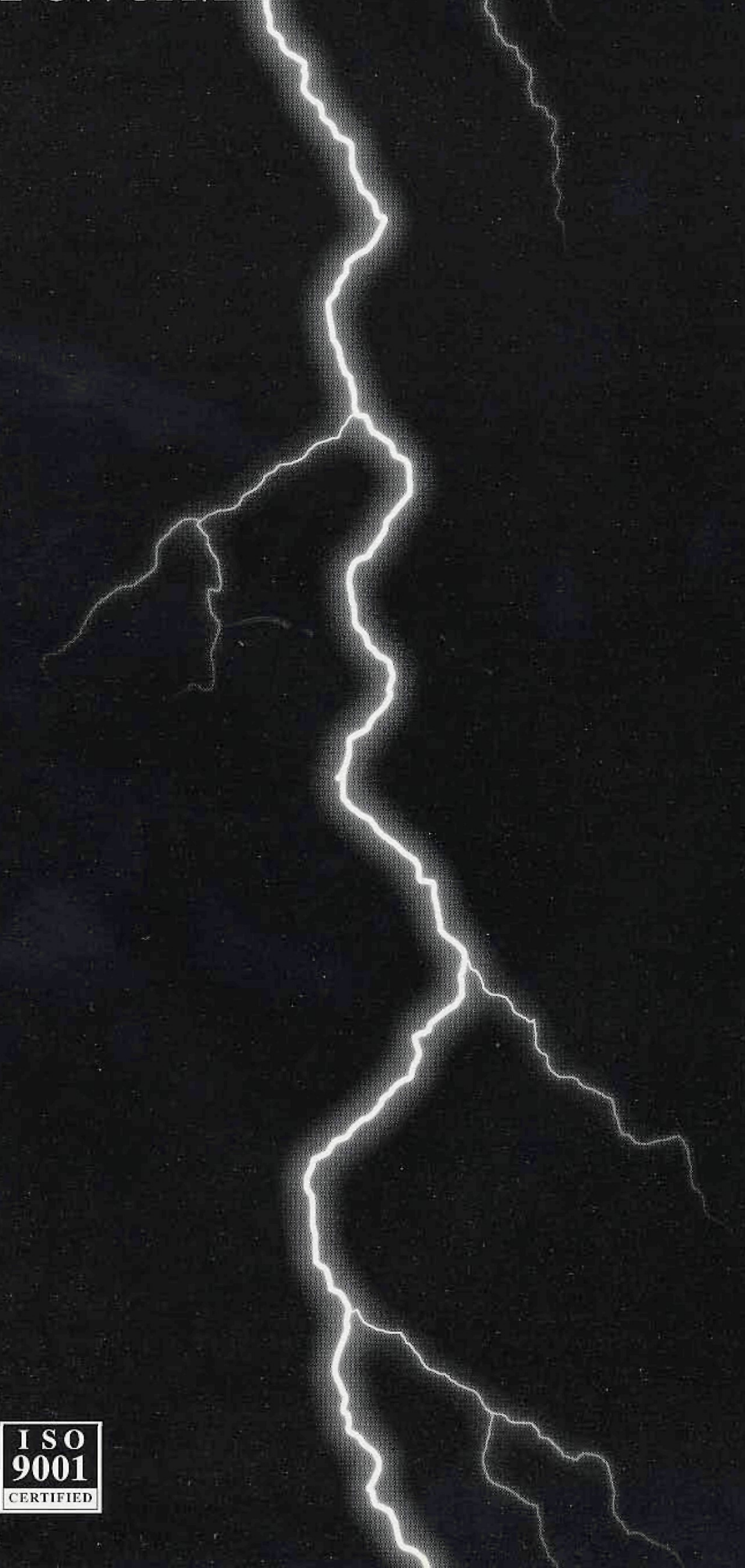

The new EDAX PowerMXTM Series adds totally new analytical capabilities to your EM analysis by integrating new Apple Macintosh PowerPC ${ }^{\text {TM }}$ technology with sophisticated EDAX data acquisition and processing know-how. The EDAX PowerMX is designed to meet the speed, expansion and imaging needs of even the most demanding analyst. Built on the new RISC-based PowerPC 600 series, the EDAX PowerMX combines the efforts of Apple, IBM, and Motorola to provide a state-of-the-art computer platform on which to run not just your EDAX designed SEM and TEM microanalysis applications, but any one of hundreds of thirdparty packages, including almost all existing Macintosh applications.

The system is built with a clear upgrade path in mind and will be state-of-the-art for years to come.

To power up your analytical capabilities, send for the EDAX PowerMX system kit today.

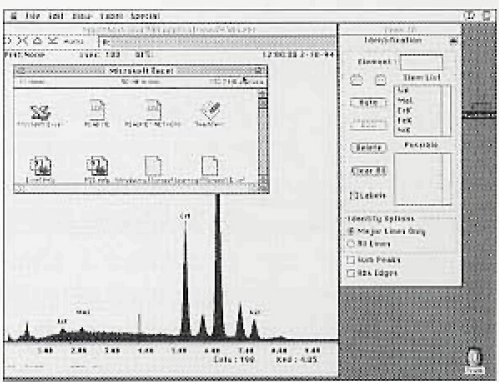

MX Spectral Display with Peak ID controls and MS EXCEL ${ }^{\text {TM }}$

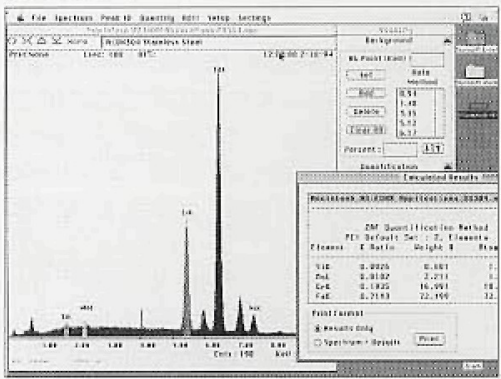

MX Spectral Display with quantitative results.

EDAX. Where knowledge makes the difference.

\section{EDAX}

\section{EDAX INTERNATIONAL}

91 Mcke Drive, Mahwah, NJ 07430

Tel. Office: 201-529-4860

Fax $201-529-3156$

Ringbaan Noord 103, P.0.Box 4144

5004 JC Tilburg, The Netherlands

Tel. 0ffice: $+31-(0)-13-364000$

Fax $+31-10) 13-356279$

13-37, Kohnan 2-chome, Minalo-ku, Tokyo 108, Japan

Tel. 0ffice: $81-3-3740-5172$

Fax: 81-3-3740-5190 